\title{
DISPLACEMENT-BASED FAILURE ANALYSIS OF METAL MATRIX SYNTACTIC FOAM
}

\author{
ISTVÁN KOZMA, DÓRA HARANGOZÓ, HAJNALKA HARGITAI \& IBOLYA ZSOLDOS \\ Department of Materials Science and Technology, Széchenyi István University, Hungary
}

\begin{abstract}
Metal matrix syntactic foams are being used more and more widely thanks to their relative density as well as their perfect energy-absorbing characteristics. Multiple studies concern the economical production of these materials - particularly, how energy absorption as a physical characteristic can be increased. Many studies examined the effects of material choice as well as cell size and wall thickness of reinforcing materials on compressive strength. However, there are only estimated models about descriptions concerning decaying processes of samples from different material composition and geometrical variation. In this study, we introduced an "in situ" examination in order to model the decaying process. We upsetted aluminium-ceramic composite foams cyclically. We reconstructed the geometry of the sample with microcomputed tomography $(\mu \mathrm{CT})$ technology and digital image processing at certain specified points of the compression test. During the complete decaying process, the process was evaluated with volume change and number of broken hollow particles, as well as elementary particle displacements, orientation and sphericity of the reinforced material.
\end{abstract}

Keywords: failure analysis, metal matrix syntactic foam, $\mu C T$, deformation analysis.

\section{INTRODUCTION}

A peculiar group of metal foams are metal foams reinforced with ceramic hollow particles. These are also known as metal matrix syntactic foams in academic literature. The two main components of metal matrix syntactic foams are the matrix material (usually technically pure aluminium, Al1050 or A199.5) or alloys of aluminium and silicon, as well as the reinforcing material [1]. The main function of these reinforcing materials (almost all of them are hollow particles) is to implement porosity within the material, and thus, to create foam structure in the total volume. Hollow particle materials are usually ceramics, but academic literature also mentions iron alloys, glass, among others, as hollow particle materials.

The main advantage of cellular materials - including metal matrix syntactic foams - is their preferential mechanical properties, alongside the relatively small density. They are used widely in car bodies and in electromechanical industry. These applications are justified by the perfect energy-absorbing characteristics and the damping effects of cellular materials. Structural rigidity and compressive strength can be set by changing the volume percentage of hollow particles of the same size. The value of volume percentage theoretically can change between $0 \%$ and $74 \%$ [2]. This data can be precisely measured with industrial radiographical devices. During our examinations, we detected a volume percentage of $64 \%$ in the sample, which was manufactured with pressure infiltration. This value equals to that of the structure of non-probable densest fitting. Thus, the location of hollow particles of almost the same diameter in the material is not regular, but the densest. Alongside volume percentage, an important characteristic of metal matrix syntactic foam structures is the size of hollow particles, their outer radius (R) and their inner radius (r) [3]. These two values must be examined together, as their ratio $(\eta=r / R)$ significantly effects the decaying behaviour of the hollow particles inside the material [4].

The most important characteristic of metal matrix syntactic foams is good compressive strength. Given the foam structure, these foams absorb energy perfectly, as the voids within the material do not collapse at the same time under the weight of an outside load, but 
separately from each other in time and space; in this way, they take up the load gradually [5]. Many studies revolve around their decaying mechanism. Most of the research examined the decaying process under quasi-static loads [2]. These experiments usually tested the effect of the combination of matrix materials and reinforcing granule materials, in relation to compressive stress. During the quasi-static examination of particle geometry of the same material composition but varying geometry, it was revealed that the tested stress values increase as the wall thickness/diameter ratio also increases [6]. An obvious relation was detected between the first maximum stress peak and the matrix material strength: the bigger the material strength, the bigger the first maximum peak of the metal matrix syntactic foam made from this material. A general testing method to track structural changes in the deforming mechanism is by evaluating cross-sectional images of the metal foam. It has been determined that under the effect of upsetting performed in quasi-static state, the hollow particles remained intact on certain parts of the metal foam; however, a break line was detectable in the cross-section of the samples [7]. The presence of the break line is the consequence of the matrix material's malleable engineering strain. In case of the material under examination, this phenomenon precedes the decaying of ceramic hollow particles used as reinforcing material. Within the break line, hollow particles broke and their voids were compacted. However, hollow particles outside the break line were much less damaged or were not damaged at all. During the examination of syntactic foam decay, Wu et al. [8] stated that hollow particles located in conical parts close to the upper and lower pressure plates which shifted their shapes only to a minor degree, stayed practically intact, they did not break. The condition of minor engineering strain for these two parts is the friction on the contact surface of the pressure plates and the samples. This hypothesis is seconded by the fact that by a single layered $\mathrm{MoS}_{2}$ lubrication, an acceptable measure of friction cannot be implemented; also, barrelling cannot be avoided. Furthermore, it was observed that in case of $90 \mu \mathrm{m}$ diameter hollow particles which give a smaller porosity $(58.1 \%)$, sample barrelling was smaller than in case of $150 \mu \mathrm{m}$ diameter hollow particles which give bigger porosity $(67.3 \%)$ [5]. Based on experiences, the authors have developed a schematic diagram about the assumed steps of decaying (Fig. 1).
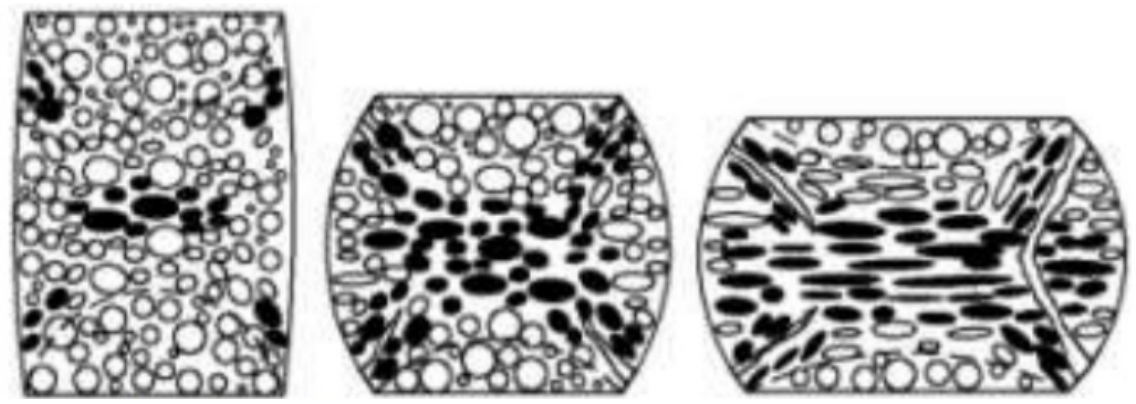

Figure 1: Schematic diagram of deformation metal matrix syntactic foam [8].

The first phase of decaying was the (shearing) decay of hollow particles found in corners in critical stress state. At the same time, hollow particle breakages occurred in the middle of the sample as well (under pressure) in the cross-sections of the weakest hollow particles. During the continuation of upsetting, damaged zones spread and a heavy barrelling occurred. This led to the detachment of material parts located in the outer volume of the sample. 


\section{MATERIALS AND TEST METHODS}

During our tests, we used cylindrical samples with aluminium (Al 99.5) matrix, reinforced with ceramics (Globocer) hollow particles $(\mathrm{R}=750 \mu \mathrm{m})$, height $\mathrm{H} /$ diameter $\mathrm{D}=1$. Pressure tests were performed according to the standard about the testing of cellular materials (DIN50134:2008). Quasi-static upsetting was performed sectionally, until a 30\% of engineering strain has been reached with the samples. The test was interrupted after $200 \mu \mathrm{m}$ of transformation in the flexible stage; and after $500 \mu \mathrm{m}$ pressure plate displacement in the flatbed and compressing stage. Sample structures were tested with microcomputed tomography $(\mu \mathrm{CT})$ technology in the starting state as well as between loads; in total, they were tested 18 times during the complete upsetting process.

X-ray Microcomputed Tomography tests were conducted with an Yxlon Modular device, with conical beam and flat panel detection. Geometric characteristics and technical parameters of the applied equipment ensured a magnification of $M=20.75$. This resulted in a digital reconstruction of samples with $19 \mu \mathrm{m}$ resolution.

During decay, change in matrix material and reinforcing material volume is negligible. However, the volumes of reinforced material voids decrease. These voids make up closed cells within the ceramic hollow particles. During shaping, cracks form on the hollow particles, then they break in certain damage zones (Figs 2 and 3). Internal hollow particle cells change in geometry as well as in topology. The decaying process was tested with these cell changes of volume, geometry and shape.

X-ray test data are the set of cubes consisting of volume and size per unit. Each of the voxels have a defined coordinate and grayvalue. We defined voxels of almost the same material with the threshold grayvalue, then we sorted them by adjacency (Fig. 4). The set of voxels defined in this way are the cell data of internal voids in the reinforced material. Data were processed with VolumeGraphics StudioMax software. In this program, we determined the geometrical and topological characteristics of each unique cell in the sampled steps of the decaying process.

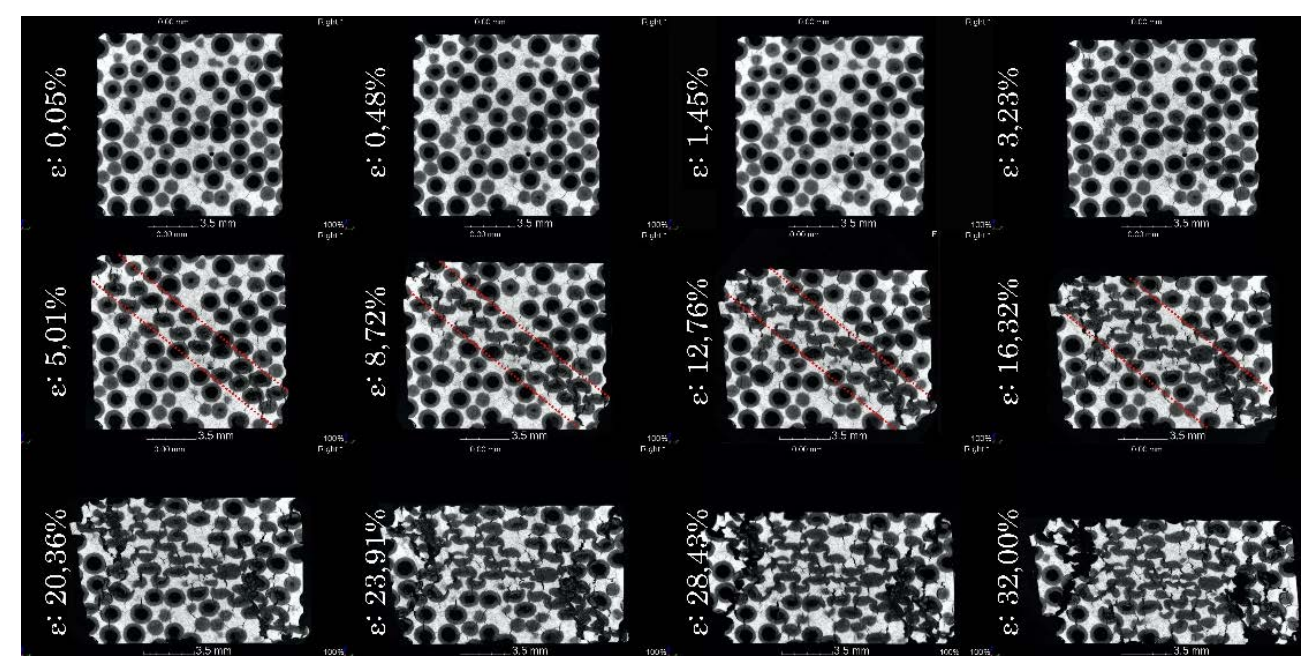

Figure 2: Structure change of metal foam on $\mu \mathrm{CT} 2 \mathrm{D}$ image slices, in relation to engineering strain. 

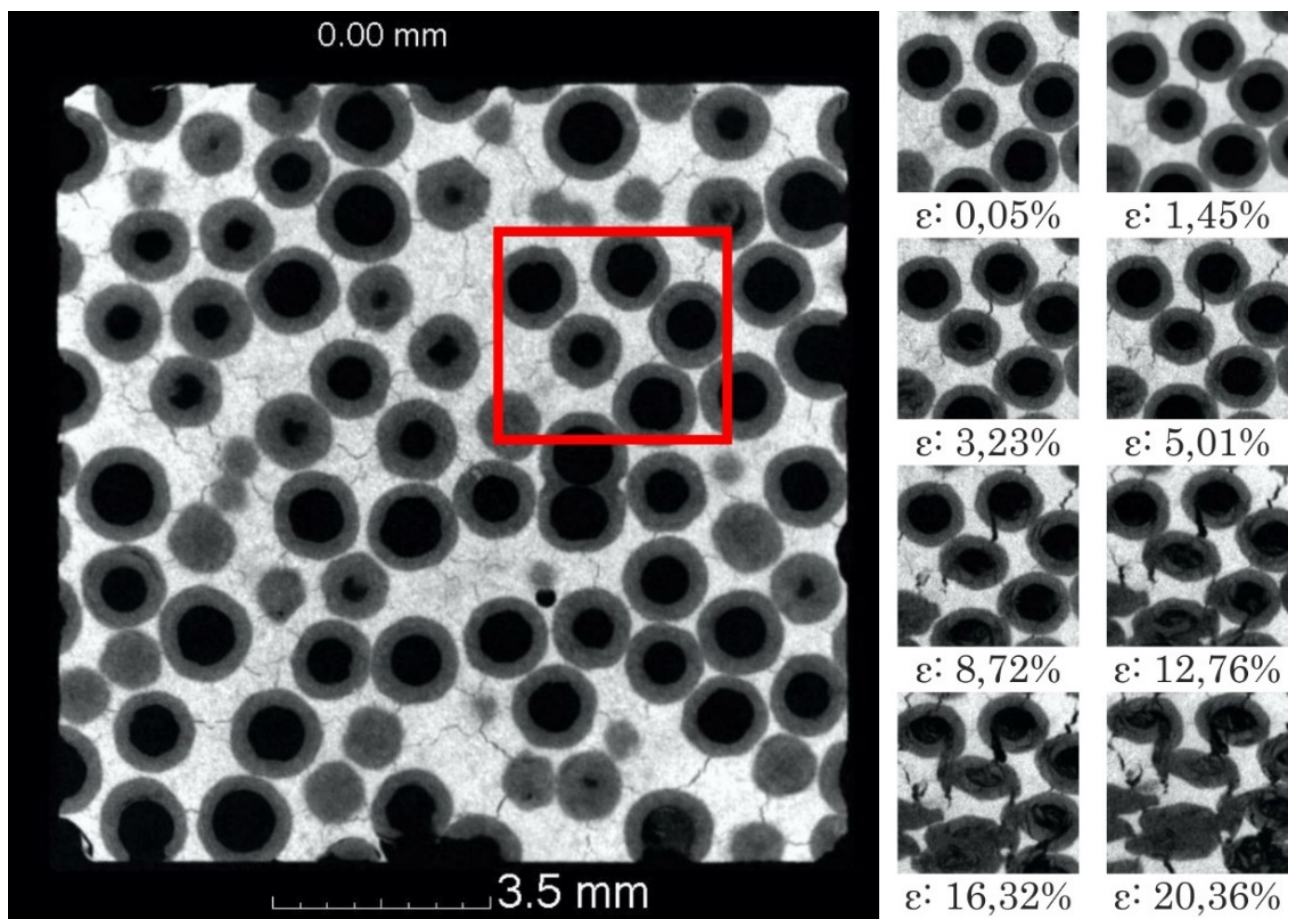

$\varepsilon: 3,23 \%$
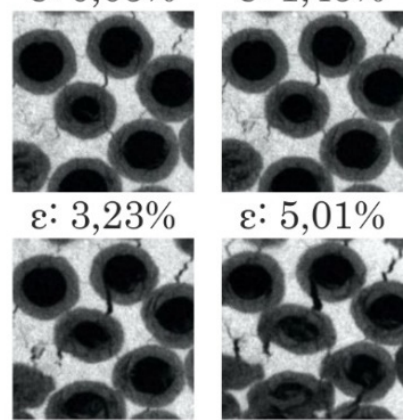

$\varepsilon: 5,01 \%$
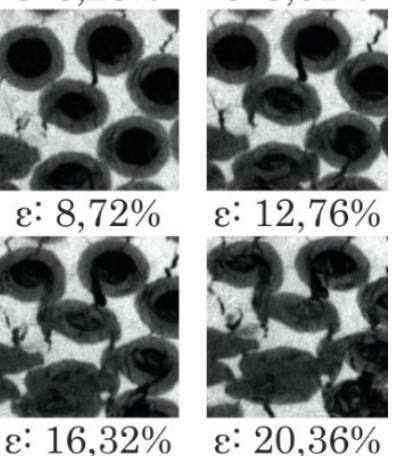

$\varepsilon: 12,76 \%$

Figure 3: Decaying on $\mu \mathrm{CT} 2 \mathrm{D}$ image slices of metal foam, in relation to engineering strain.

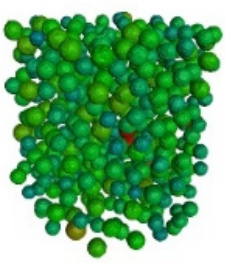

Air void

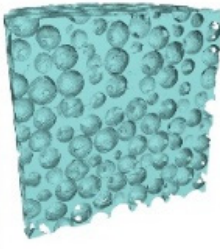

Matrix

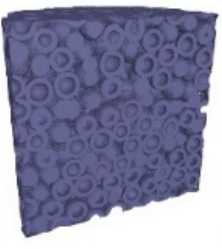

Particle shell

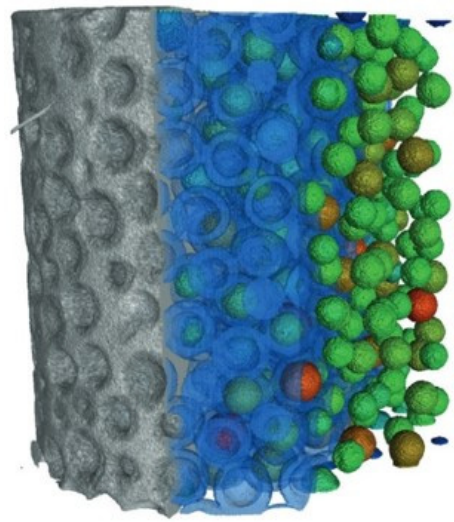

(b)

Figure 4: Consisting elements of metal foam samples in relation to density.

Data were saved in a text file which includes $\mathrm{x}, \mathrm{y}$ and $\mathrm{z}$ coordinates for the hollow particle voids, as well as their respective volume and diameter values, their spherical shape factors, and the number of hollow particle voids. Data assigned together were evaluated in GOM 
Inspect software. Small, consecutive engineering strains induced slight displacements. Each coordinate of the consecutive states was fitted to the coordinates of the next phase state, based on the closest adjacent algorithm. Search distance was maximized in the value of the average hollow particle radius; thus we ensured the accuracy of the searching algorithm. We assigned an identifier to the hollow particle coordinates of the starting sample (Fig. 4(b)). This identifier marked that certain unique hollow particle during the whole transformation process, including its volume and diameter data (Fig. 5).

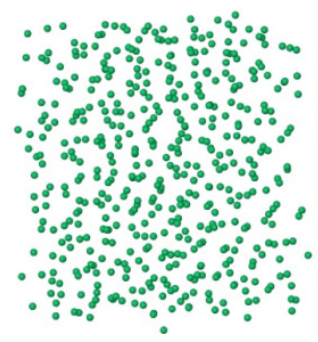

(a)

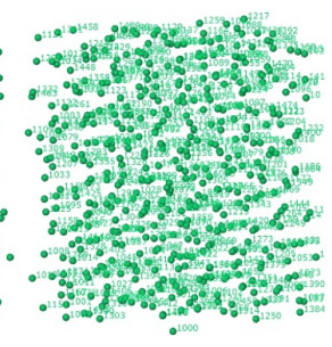

(b)

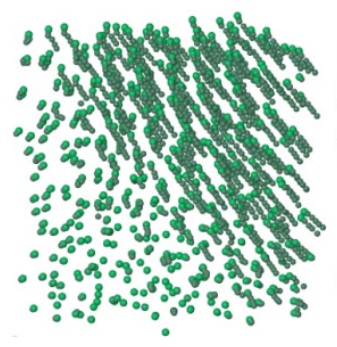

(c)

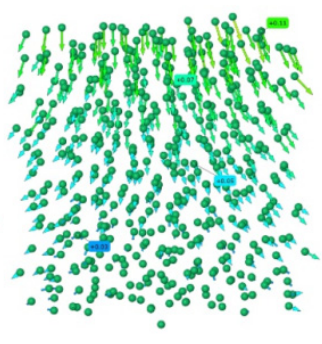

(d)

Figure 5: (a) Hollow particle coordinates and (b) their identifiers, as well as Hollow particle displacements (c) as point cloud and (d) in vectorial display, in relation to engineering strain.

\section{RESULTS}

During the metal foam sample decay tests, we evaluated sectional images of X-ray tests as well as coordinate, volume and diameter data of unique hollow particle voids at the same time.

We inserted volume change data of reinforcing material into the push chart (Fig. 6). In the first phase of engineering strain, volume changes confirm literature references. Radius ratio of the hollow particles $(\eta)$ affects volume change during the transformation process. Under load, the volume of hollow particles forming the reinforced material of samples - due to their radius direction - increase until the first stress peak of the transformation. Volume increase can be detected until an engineering strain of $2 \%$; then, near the stress peak, it started to decrease steeply. The intensity of volume decrease changed at $4 \%$ engineering strain, and it showed near linear decrease in the flatbed stage.

The metal foam is significantly damaged during the transformation, within the $2 \%-5 \%$ engineering strain interval. After reaching the maximum stress, cracks form on ceramic hollow sphere walls under further load, and a $45^{\circ}$ angle shear plane forms on the transformation direction. This range at the 2\%-5\% engineering strain load can be unequivocally determined based on both the sectional images and the volume change of samples. Along the $45^{\circ}$ plane, the damage zone thickens under further pressure.

At $5 \%$ engineering strain it thickens to such a measure that a glide plane forms in this section of the sample. During further loading, the sample compresses in the complete volume of the test sample. Under the flatbed stage of the push chart, volume decrease is almost linear; it its intact after 30\% engineering strain, defragmented hollow particles cannot be detected and hollow particle void volumes decrease to the detection threshold value. 


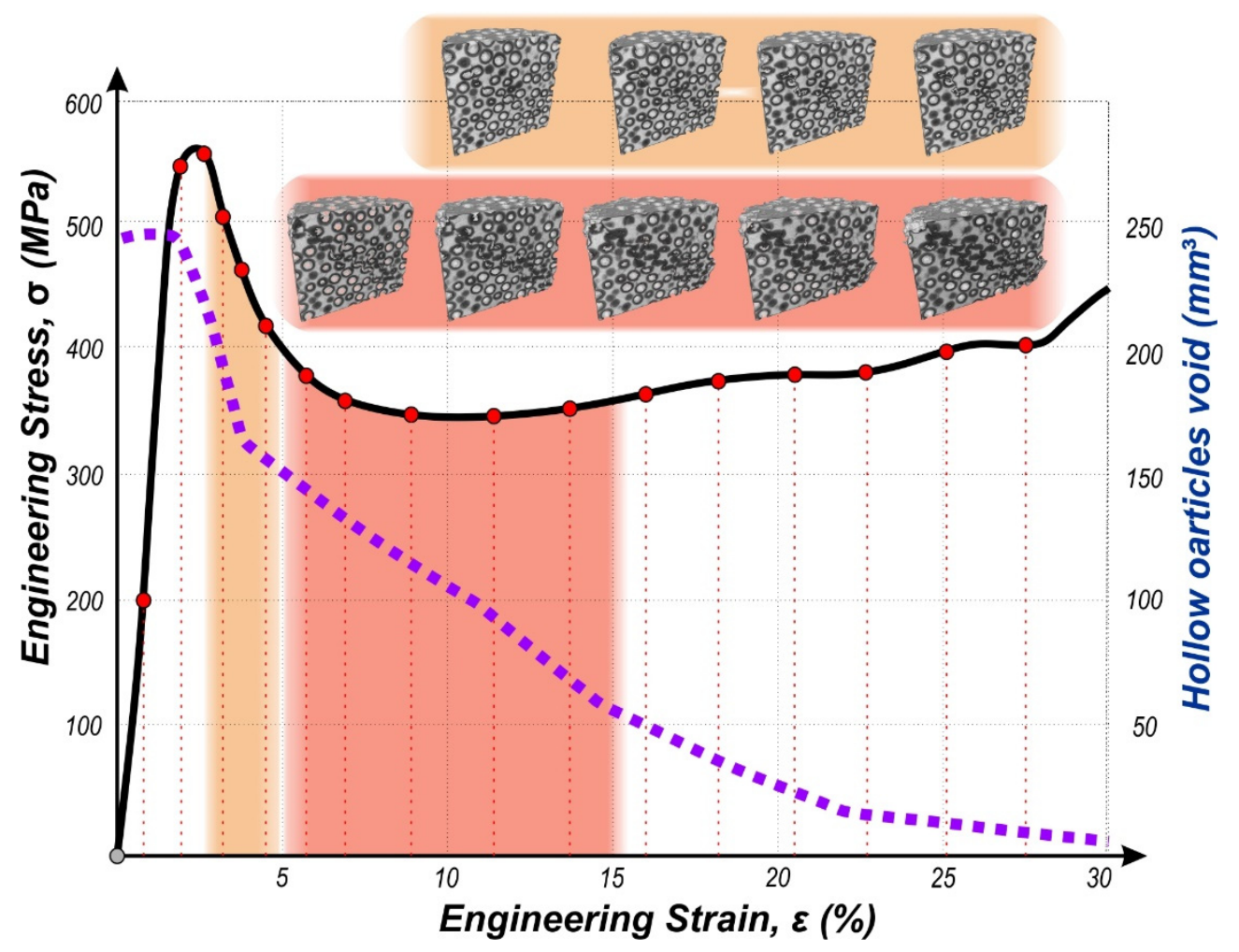

Figure 6: Push chart of tested samples and volume changes of hollow particle voids.

Hollow particle displacements induced by loads are depicted as vectors. The starting point of vectors is always the given, marked hollow particle gravity centre of the untransformed sample; the gravity centre of given state and position of the other hollow particle under the same identifier (Fig. 5). By this method, we determined not only the direction of displacement, but its size as well.

Our results show that, in the first stage of decaying which lasts up to the stress maximum of the push chart, engineering strain of the metal foam sample shows a similar process up to $\sim 2 \%$ engineering strain as that of homogeneous materials (Fig. 7). Samples are barrelling, displacement vectors are small and they point to the lateral surface direction of the sample, almost equally from the axis.

The behaviour of metal foam can be explained with the malleable engineering strain of the aluminium matrix. At this stage, hollow particles did not take up critical load. However, at $2 \%$ engineering strain, there are detectable cracks in the matrix material (Fig. 3). Under further transformation, decaying starts on the hollow sphere wall, in thickness direction. At this stage, up to $\sim 5 \%$ engineering strain, a $\sim 45^{\circ}$ angle break line forms in the loading direction. Hollow particles right next to the line start to break, then they completely detach. Hollow particle void volumes start to decrease significantly; this change correlates with the curve of the push chart. 

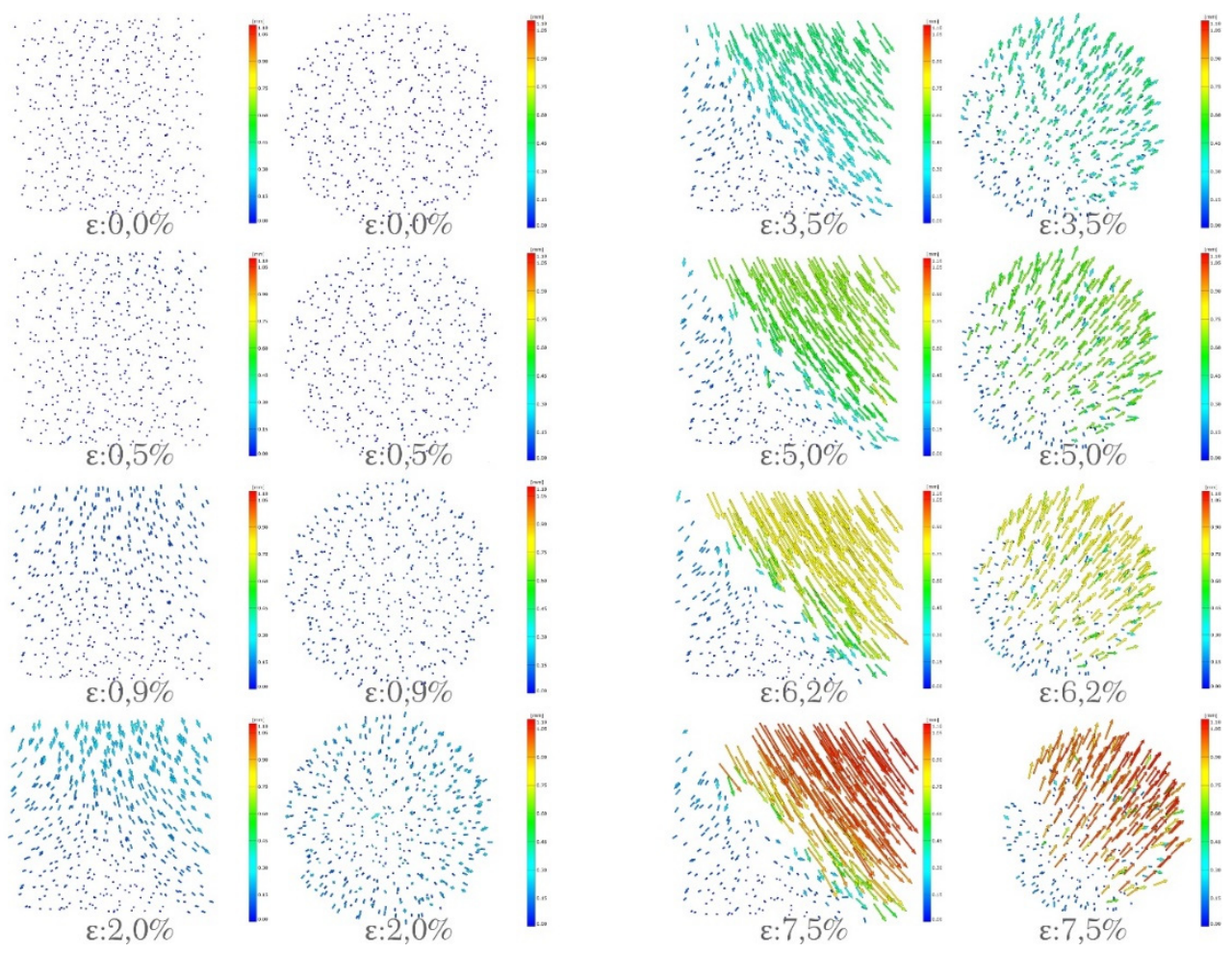

Figure 7: Hollow particle displacements, in relation to engineering strain.

Characteristics of displacement vectors also definitely change. The angle close to $45^{\circ}$ with the load direction borders two different displacement structures (Fig. 8). In one of the segments, the barrelling characteristic remained with small homogeneous displacements. However, in the other area of the sample, big dislocations occur, usually in the same direction. Based on the vectorial map, the break line is also a shear plane, which is also a gliding plane during further transformations. Increasing the $5 \%$ engineering strain, the decaying process becomes homogeneous. By continuing upsetting, the shear plane thickens into a break line; the inner volume of hollow particles located on the line drastically decreases, then the line thickness increases further during decay; in the starting plane, the inner volume of the hollow particles reduces to a level where they no longer can be detected. Up to $20 \%$ engineering strain, hollow particles remote from the gliding plane stay intact. Above $20 \%$ engineering strain, decaying spreads to the whole sample. Fragmentation, decaying and volume change of hollow particles significantly increases in other segments of the sample as well.

We evaluated hollow particle displacements as only $\mathrm{X}, \mathrm{Y}$ and $\mathrm{Z}$ directional components as well. Results have confirmed the previously described processes. The Z-directional displacement field shows the expected result. The largest displacement is on the load lateral surface; the length of vectors decreases towards the resting jaw. X- and Y-directional evaluation confirms the behaviour typical in case of homogeneous materials in the starting phase of transformation. Up to $2 \%$ engineering strain, displacements point symmetrically to 
the given tested ( $\mathrm{x}$ and $\mathrm{y}$ ) planes, or to the sample axis with the same vector size, only to the opposite direction.

Loading the samples further, the vector extent (that is, displacement) increases significantly. Next to increase, it shows an asymmetric image, as opposed to the starting symmetrical plane. It can be observed that this plane is not parallel to the load direction anymore, not even by increasing the transformation; it makes an angle with the load direction. The angle difference constantly grows above $2 \%$ engineering strain. Close to a $3.5 \%$ engineering strain, it makes a $\sim 12^{\circ}$ angle with the load direction (Fig. 8); above $4.5 \%$ engineering strain, it makes a $\sim 30^{\circ}$ angle (Fig. 9(b) and (c)); and at the measurement point above $5 \%$ engineering strain, it makes a $45^{\circ}$ angle. Under the effect of shear power, the glide plane happens within the $2 \%-5 \%$ engineering strain range, based on hollow particle displacement.

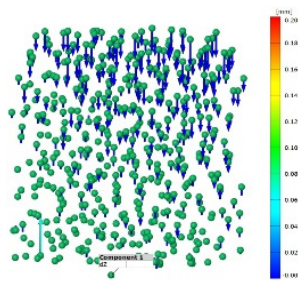

(a)

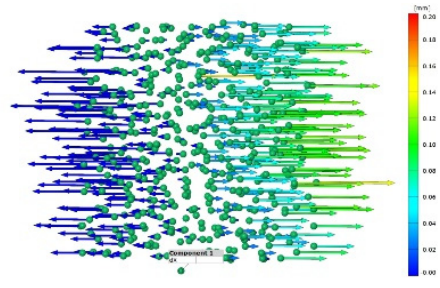

(b)

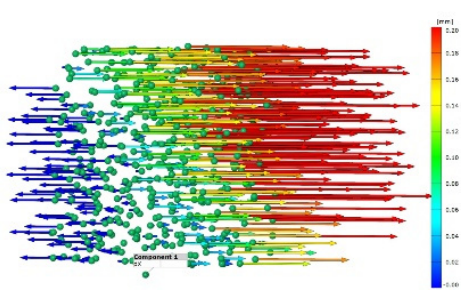

(c)

Figure 8: Displacement of hollow particles in the direction of transformation $(\mathrm{Z})$ : (a) Above $2 \%$ engineering strain. Displacement of hollow particles, perpendicularly to the transformation direction (X): (b) Above 2\% engineering strain; (c) Above 5\% engineering strain.

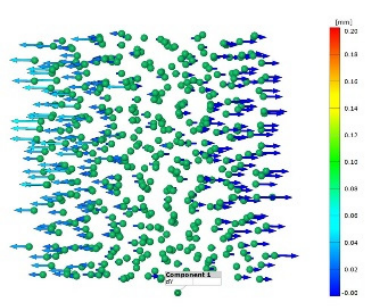

(a)

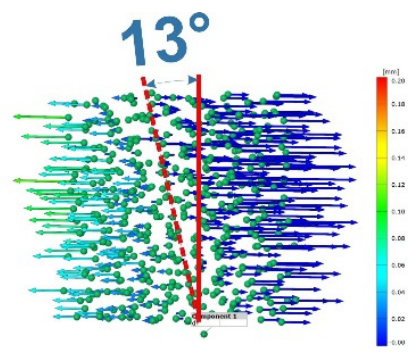

(b)

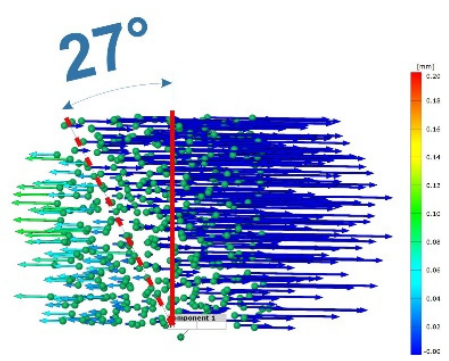

(c)

Figure 9: Displacement of hollow particles, perpendicularly to the transformation direction (Y): (a) Above 2\% engineering strain; (b) Above 3.5\% engineering strain; (c) Above 5\% engineering strain.

The displacement histogram depicted in Fig. 10 also supports this. This figure quantitatively summarizes the numerical data of previously, graphically presented displacement vectors, in relation to engineering strain. On the $\mathrm{X}$ axis of the diagram, displacement values under given load can be read; on the $\mathrm{Y}$ axis, the number of displaced hollow particles can be read. It can be seen from the results that up to $2 \%$ engineering strain, 
the number of hollow particles changing position under load gradually increases; however, after further load, the quantitative factor does not change substantially, it becomes steady. At the same time, the distribution of displacement sizes during transformation supports earlier measurements and statements. The "barrelling", a homogeneous displacement characteristic, can be detected up to $2 \%$ engineering strain. The curve of the histogram does not show any local peaks; the size of displacements can be viewed as normally distributed. At 3.5\% engineering strain, the homogeneity of the displacement field ceases to exist, 2 local peaks can be detected. The area under the two peaks - meaning, the number of hollow particles corresponding to given displacement measures - is approximately of the same magnitude. This similarity indicates two identical volumes in the same way as the linearly measurable distance between local peaks.

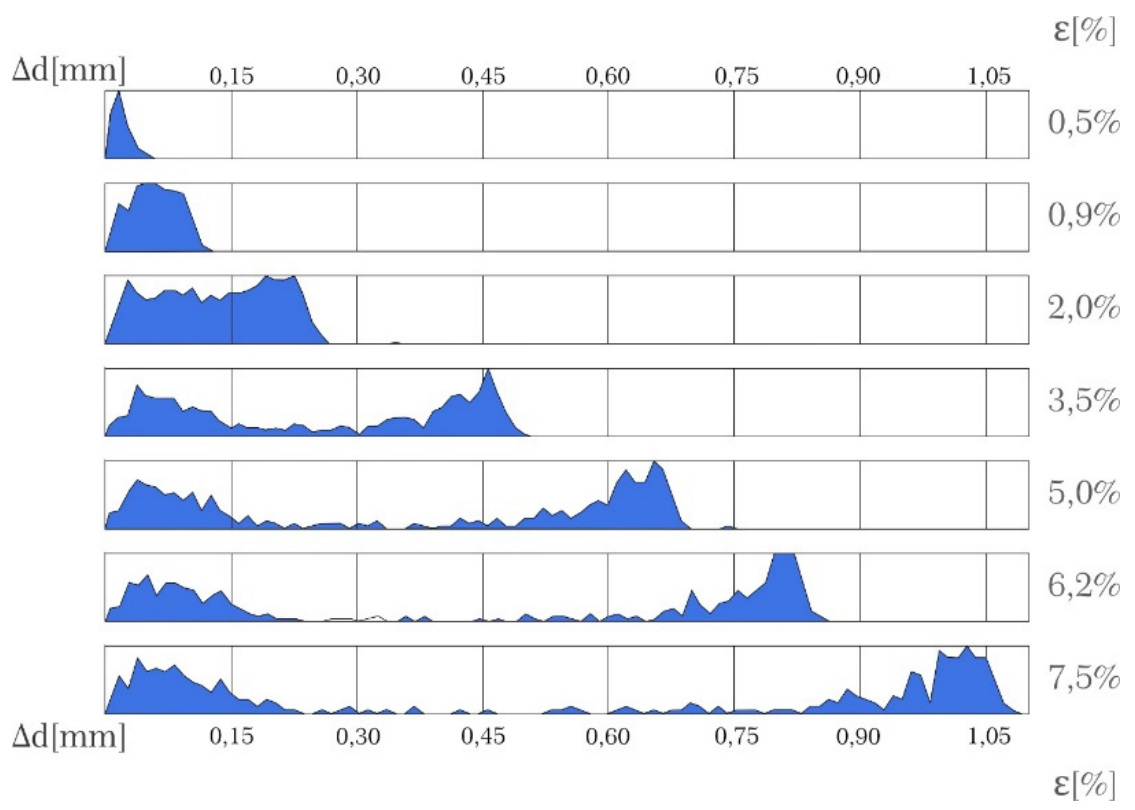

Figure 10: Displacement histograms in relation to engineering strain.

In Fig. 11, we can see the hollow particle displacement vectors of a sample above 2\% engineering strain. We edited the excitation lines of vector displacement next to the figures - this is the characteristic referring to as "barrelling". In Fig. 11(b), the vector direction does not show only the engineering strain; arising forces can also be deduced based on the orderliness of directions. Based on this, it can be demonstrated that at this phase of transformation, the metal foam shows engineering strain typical to that of homogeneous materials; it deforms align a pressure cone. By increasing the load further, the characteristic shear plane forms.

Next to the displacement of gravity centres, we examined hollow particle surface deformation during transformation process. Volume data from microcomputed tomography scan were converted into surface models, then we evaluated the differences. In Fig. 12(a), the detail shows the starting geometry (blue) as well as geometry respective to $5 \%$ engineering strain (grey). In Fig. 12(b) and (c), details show differences between the two geometries, from different viewpoints. Red indicates additional material; blue indicates 
material loss. The evaluation confirms earlier deductions: parts can be localized in the sample structure where deformation cannot be detected. The big differences between geometries are detectable above the plane closing $45^{\circ}$ degree with the axis. The detected surface differences usually do not show axial deformations either. Local geometry differences also occur in $45^{\circ}$ degree direction.

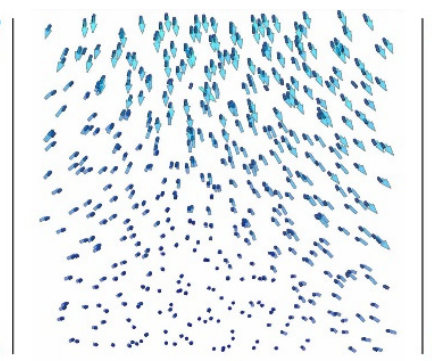

(a)

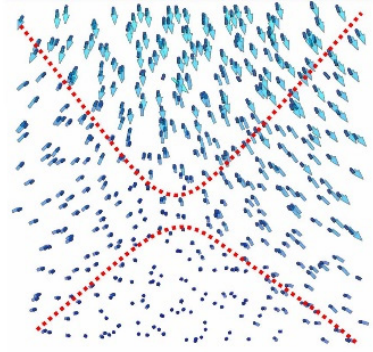

(b)

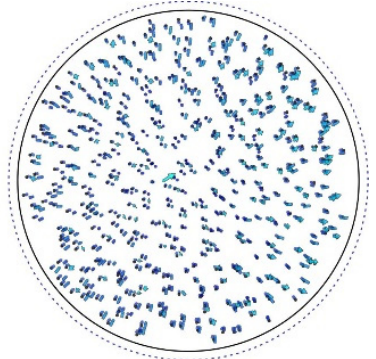

(c)

Figure 11: Hollow particle displacement vectors after 2\% engineering strain histograms in relation to engineering strain.

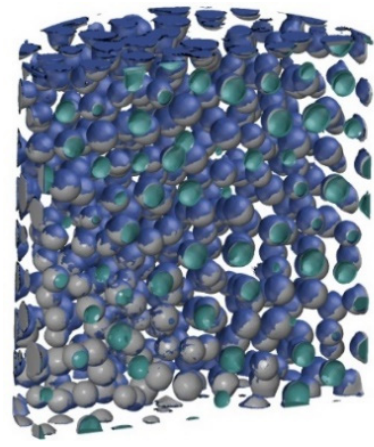

(a)

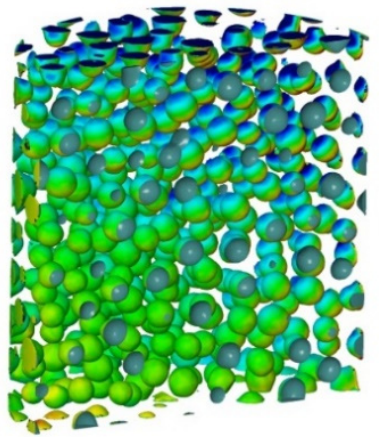

(b)
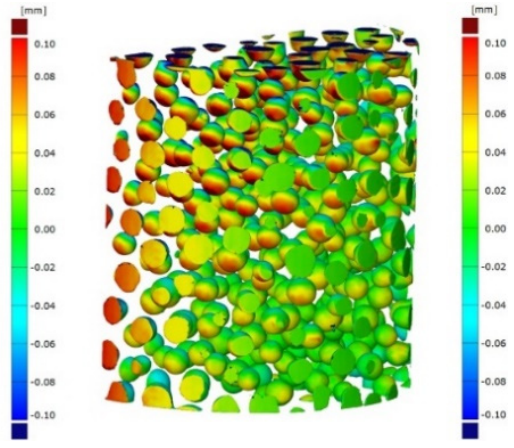

(c)

Figure 12: Hollow surface differences between the two geometries.

We have chosen 5 typically located hollow particle geometries from global surface data (Fig. 13), and we evaluated their local characteristics further. The SPH_A hollow particle is marked perpendicularly to the shear plane, right next to the upper pressure plate; the SPH_B hollow particle is marked on the decaying line border, closer to the upper pressure plate; the SPH_C hollow particle is marked in the centre of the decaying zone; the SPH_D hollow particle is marked on the decaying line border, closer to the lower pressure plate; and the SPH_E hollow particle is marked right next to the lower pressure plate, in the segment which was transformed the least.

Hollow particle displacements are presented in the diagram of Fig. 14. Displacement values can be stated linear in case of each hollow particle in relation to engineering strain; however, the scale depends on their position. There is no detectable, significant difference between the 2-2 hollow particles above and under the break line. 

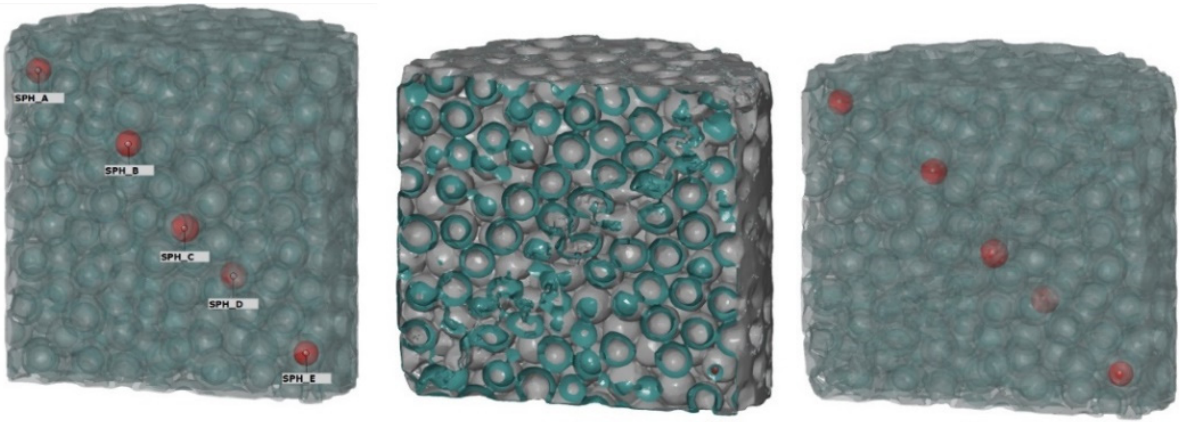

Figure 13: Individually evaluated hollow particles on the microcomputed tomography reconstruction.

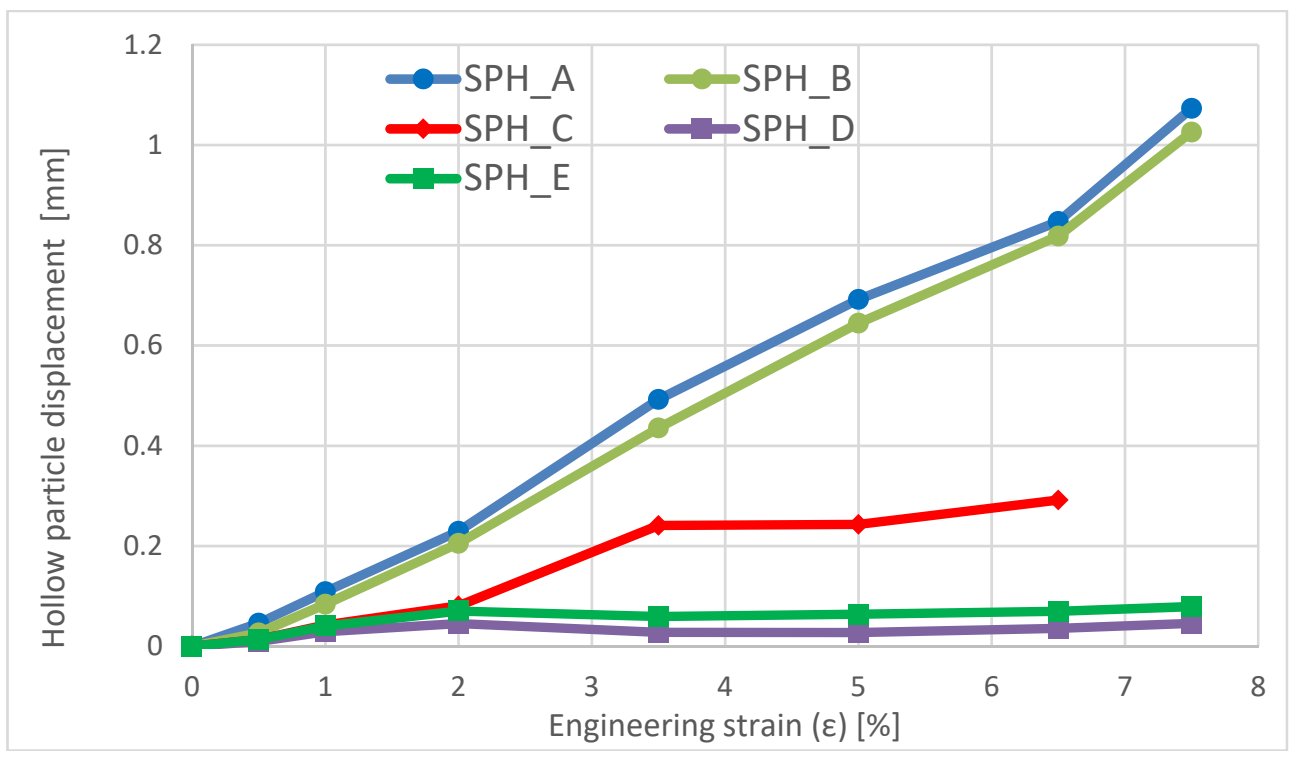

Figure 14: Local hollow particle displacements, in relation to the engineering strain.

During transformation, hollow particles deform, their shape characteristics change. The value of this phenomenon was characterised by the sphericity factor, based on the equation below

$$
\Psi=\frac{\pi^{\frac{1}{3}}\left(6 V_{p}\right)^{\frac{2}{3}}}{A_{p}} .
$$

Measured values are presented in the diagram of Fig. 15. In the sphericity characteristic, the biggest difference was measured on the hollow particle marked in the break line. Change is depicted on the image series of Fig. 16. The deformation scale of further hollow particles is determined by their positions. Deformation was the biggest near the upper pressure plate; however, significant deformation cannot be detected near the lower pressure plate. 


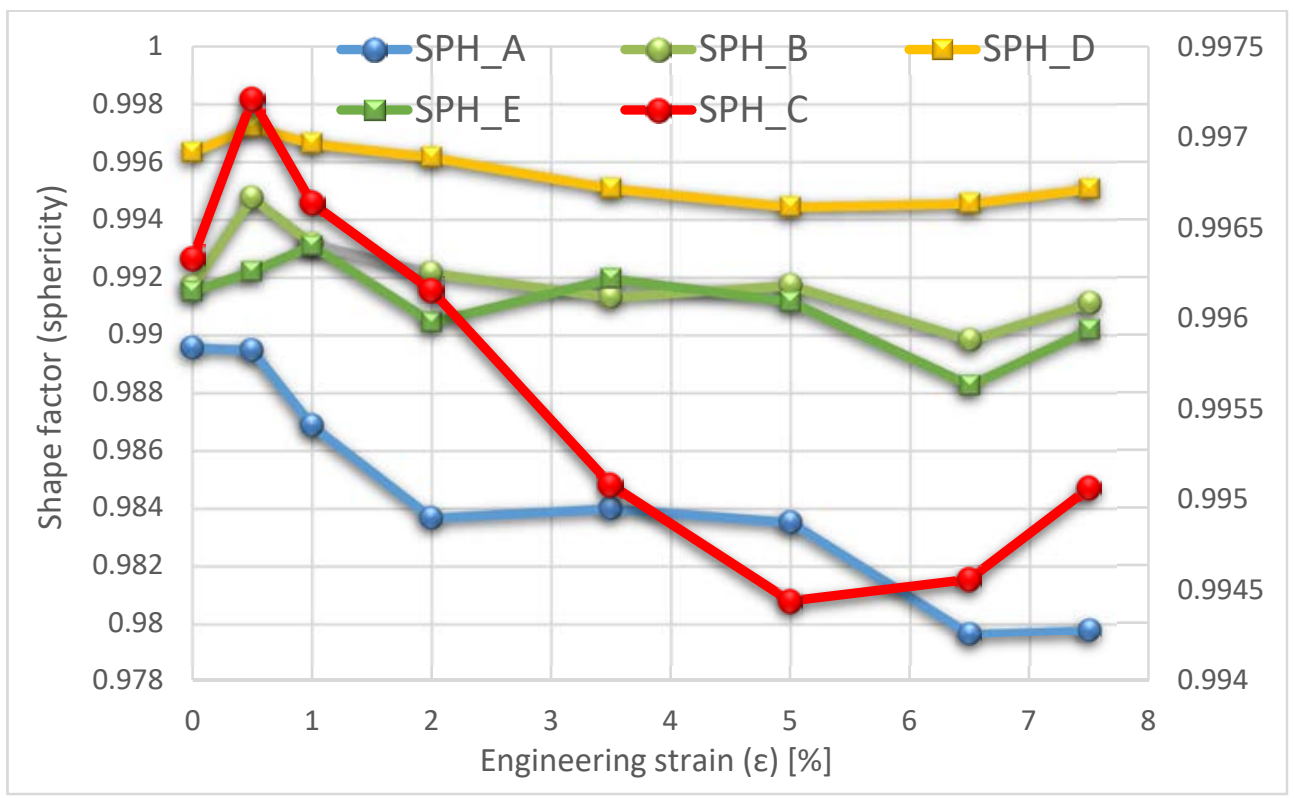

Figure 15: Change of sphericity factors in relation to transformation.

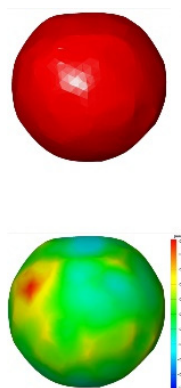

$\varepsilon: 0 \%$
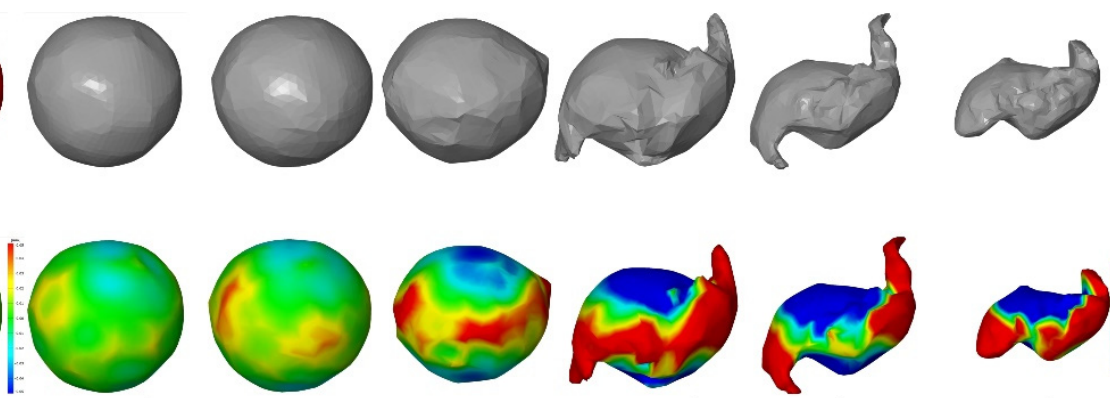

$\varepsilon: 2.0 \%$

$\varepsilon: 3.5 \%$

$\varepsilon: 5.0 \%$

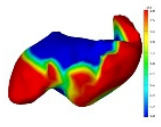

$\varepsilon: 6.5 \%$

Figure 16: Deformation of hollow particle in the centre of the decaying line, in relation to engineering strain.

\section{CONCLUSIONS}

In this paper, we upset the metal matrix syntactic foam samples in stages. In order to model the decaying process, we reconstructed the geometry of the sample with X-ray Microcomputed Tomography technology and digital image processing at certain specified points of the compression test. During the complete decaying process, the process was evaluated with volume change, as well as elementary particle displacements, orientation and sphericity of the reinforced material.

During our tests, we concluded that at $2 \%$ engineering strain, there are detectable cracks in the matrix material. Under further transformation, decaying starts on the hollow sphere wall, in thickness direction. At this stage up to $\sim 5 \%$ engineering strain, a $\sim 45^{\circ}$ angle break 
line forms on the load direction. Hollow particles right next to the line start to break, then they completely detach. Hollow particle void volumes start to decrease significantly; this change correlates with the curve of the push chart. We also evaluated the decaying process with hollow particle displacements. We concluded that the angle of $45^{\circ}$ with the load direction borders two different displacement structures. In one of the segments, the barrelling characteristic remained with small homogeneous displacements. However, on the other area of the sample, big dislocations occur, usually in the same direction. Based on the vectorial map, the break line is also a shear plane, which is also a gliding plane during further transformations. Increasing the $5 \%$ engineering strain, the decaying process becomes homogeneous. By continuing upsetting, the shear plane thickens into a break line; the inner volume of hollow particles located on the line drastically decreases, then the line thickness increases further during decay; in the starting plane, the inner volume of the hollow particles reduces to a level where they no longer can be detected. Up to $20 \%$ engineering strain, hollow particles remote from the gliding plane stay intact.

\section{ACKNOWLEDGEMENTS}

We acknowledge EFOP-3.6.1-16-2016-00017 Internationalization, initiatives to establish a new source of researchers and graduates, and development of knowledge and technological transfer as instruments of intelligent specializations at Szechenyi University.

\section{REFERENCES}

[1] Orbulov, I.N. \& Majlinger, K., Microstructure of metal-matrix composites reinforced by ceramic microballoons. Materiali in Tehnologije, 46(4), pp. 375-382, 2012.

[2] Orbulov, I.N. \& Májlinger, K., Compressive properties of metal matrix syntactic foams in free and constrained compression. JOM, 66(6), pp. 882-891, 2014.

[3] Balch, D.K. et al., Plasticity and damage in aluminum syntactic foams deformed under dynamic and quasi-static conditions. Materials Science and Engineering: A, 391(1), pp. 408-417, 2005.

[4] Vesenjak, M. et al., Impact behavior of composite hollow sphere structures. Journal of Composite Materials, 43(22), pp. 2491-2505, 2009.

[5] Gupta, N. \& Ricci, W., Comparison of compressive properties of layered syntactic foams having gradient in microballoon volume fraction and wall thickness. Materials Science and Engineering: A, 427(1), pp. 331-342, 2006.

[6] Gupta, N. \& Rohatgi, P.K., Metal Matrix Syntactic Foams: Processing, Microstructure, Properties and Applications, DEStech Publications, 2014.

[7] Zou, L.C. et al., Compression deformation behaviour of aluminium matrix syntactic foams under quasistatic loading: In situ SEM compressive tests. Materials Research Innovations, 18(sup4), pp. S4-536-S4-539, 2014.

[8] Wu, G.H. et al., Compression behaviors of cenosphere-pure aluminum syntactic foams. Scripta Materialia, 56(3), pp. 221-224, 2007. 